\title{
Research on Boost-Buck High-efficiency Semiconductor Lighting Driving Based on OCC Feedforward Technology
}

\author{
Renbo $\mathrm{Xu}^{1.2}$ and Youhui Zou ${ }^{1}$ \\ ${ }^{1}$ School of electrical and information Engineering, Nanchang Institute of Science \& Technology; \\ ${ }^{2}$ Physics and Microelectronics Institute, Central South University
}

Keywords: Semiconductor lighting; CUK; OCC feedforward; Efficient and stable

\begin{abstract}
Semiconductor lighting has the characteristics of energy saving, high efficiency, long life and environmental protection. It is the product of "green lighting" and semiconductor luminescence technology, and CUK converter is widely used in semiconductor lighting drive. In this paper, the main features of CUK main circuit are discussed, and a new CUK high power and high efficiency semiconductor lighting control scheme is proposed, that is, the CUK drive system controlled by OCC feedforward technology. Then, the author makes a deep research on its work dynamics, such as SIMULINK and SIMPOWERSYSTEMS and other tools simulation analysis, and the corresponding relationship expression of its system dynamic performance is derived to achieve that the system has the characteristics of high efficiency, fast response, strong anti-interference and so on.
\end{abstract}

\section{Introduction}

CUK converter is also known as Boost-Buck converter, as shown in Fig. 1. It is one of the best topologies in semiconductor lighting drives, and is widely used [1-6], with both boost and buck output, maintaining the continuity of Boost-type converter input current with a wide input voltage range. It can easily achieve boost or buck by changing the duty cycle of the switch. Through serial in two inductors, the exchange ripple wave value becomes smaller, in practice, electromagnetic interference (EMI) filter does not need to be added. The drive system is small and the voltage is stable. Based on the traditional control, this paper presents a new scheme, OCC feedforward drive system.

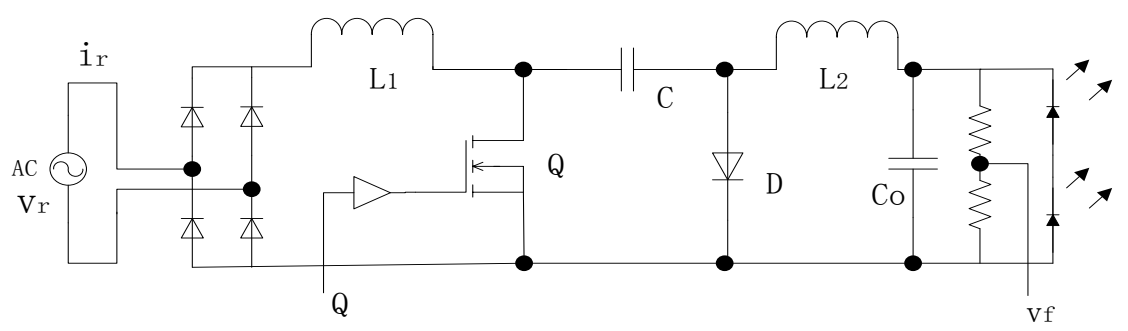

Figure 1. Schematic diagram of CUK convertor

\section{Basic Ideas and Implementation Scheme of OCC Feedforward Control}

At present, most of the drive control is to linearize the obtained model equation firstly, and then use a feedback loop to control [7-10]. The OCC control technology is shown in Fig. 2. However, due to the dynamic characteristics of the filter, the controlled output voltage will be disturbed, and its anti-load disturbance capability is weak, and the power factor of the whole converter may decrease at light load. In view of these problems, this paper proposes OCC feedforward control 
improvement strategy, as shown in Fig. 3.

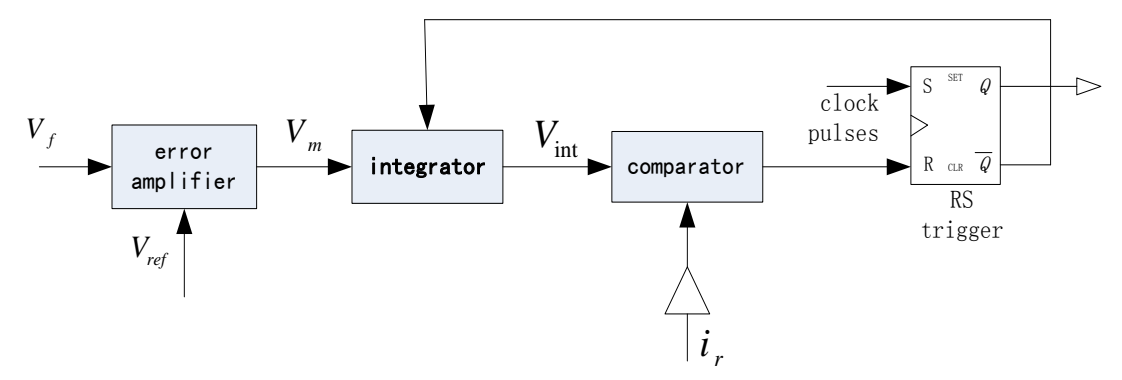

Figure 2. Control strategy of CUK convertor for traditional OCC

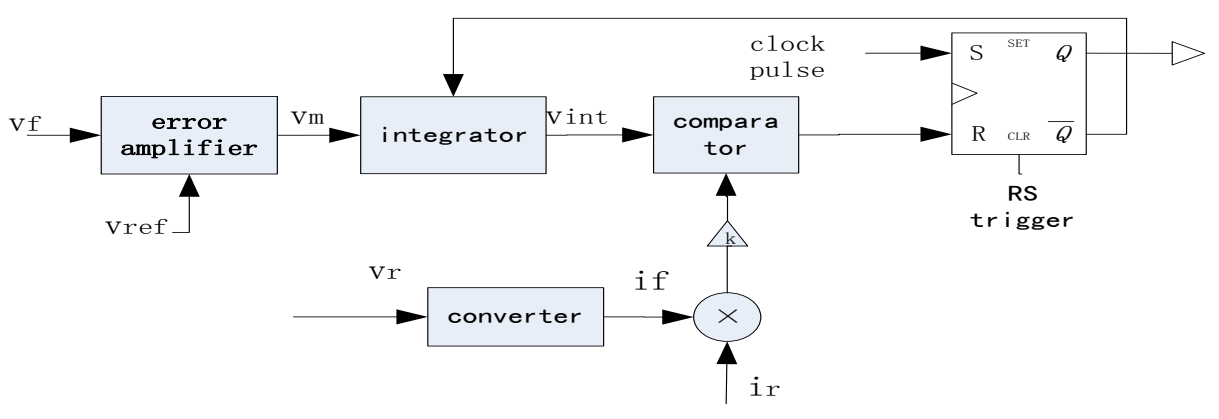

Figure 3. Feedforward control improvement strategy of CUK convertor based on OCC

As can be seen from the figure above, the sampling voltage is taken from the system output voltage and a feedback signal at input terminal is added. OCC feedforward control improvement strategy using voltage feedforward control technology, the input voltage $V_{r}$ is converted to get the synchronous current $i_{f}$ signal, which is added with the input current $i_{r}$ signal and sent to the comparator with the integrator output signal together, generating switch tube driver signal through RS trigger. The control strategy is changed from sampling of converter for the input voltage to the current signal $^{i_{f}}$, which can effectively reflect the disturbance of the input voltage. The disturbance of input voltage can be suppressed by the adjustment of single cycle. If the input voltage $v_{r}$ has a pulse transition, the current signal $i_{r}$ obtained by the converter will produce a pulse ripple, and the output voltage will become larger, the feedback voltage signal $V_{f}$ will fluctuate, which will increase the integral slope of the integrator, integration time will decrease, the duty cycle will be reduced accordingly, and the voltage has been stable output, to ensure a good anti-interference ability.

Analysis under the conditions of the previous assumption, the working waveform is shown in Fig. 4. Set a converter conversion rate $k_{b}$, that is $i_{f}=v_{r} k_{b}$, the following equation can be got by the working waveform:

$$
v_{r}=L_{s} \frac{\Delta i_{r}}{T_{S}}+\left(i_{r}+i_{f}\right) \frac{V_{f}}{V_{m}}
$$


Where $L_{s}$ is equivalent inductance, $\Delta i_{r}$ is the peak current variation within single cycle.

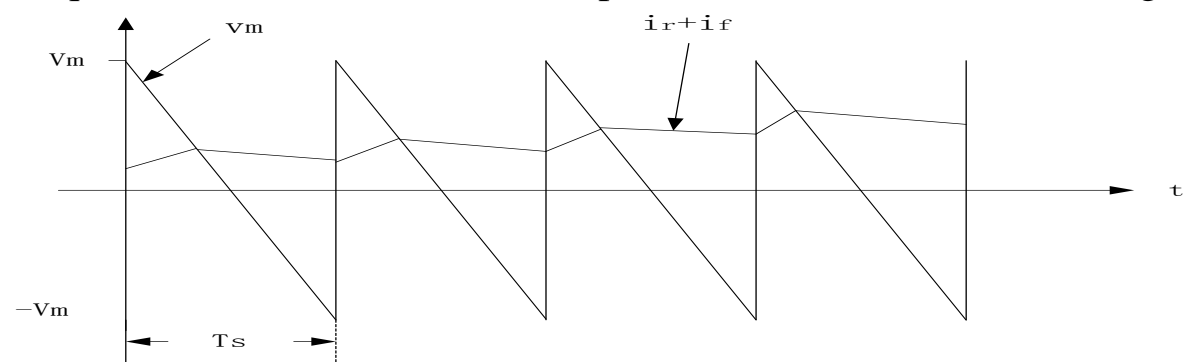

Figure 4. Work waveform based on OCC feedforward control improvement strategy

The equation below can be obtained combining $i_{f}=v_{r} k_{b}$ and the assumed conditions aforesaid:

$$
v_{r}\left(1-\frac{V_{f}}{V_{m}} k_{b}\right)=i_{r} \frac{V_{f}}{V_{m}}+L_{s} \frac{d i_{r}}{d t}
$$

Which can simplified as:

$$
i_{r}=v_{r}\left(1-\frac{V_{f}}{V_{m}} k_{b}\right) / \frac{V_{f}}{V_{m}}+j \omega L_{s}
$$

From the equation above, the introduction of convertor conversion rate $k_{b}$ reduces the effect of error amplifier output on the entire system, also achieves precise adjustment of the output voltage to overcome the deficiency of traditional converter and improve the performance of system in light load characteristics and power factor, as well as improves the anti-load disturbance capability. If the output feedback voltage $V_{f}$ is much larger than the error signal, and the error signal is equal to the conversion rate, the impact of error signal can be ignored, that is the effect of error signal on the entire drive system is completely eliminated.

\section{Simulation Results and Analysis}

In order to verify the validity of feedforward control improvement strategy of CUK converter of OCC, SIMULINK and SimPower Systems tools in MATLAB software are used to set up CUK main circuit simulation model. The input voltage is sinusoidal $220 \mathrm{~V}, 50 \mathrm{~Hz}$ AC voltage, the load LED resistance is $20 \Omega$, the output voltage is set to $20 \mathrm{~V}$, the capacitance $C$ is $0.1 \mathrm{uF}$, the inductance $L_{1}$ is $2.7 \mathrm{mH}$, the output capacitance $C_{O}$ is $470 \mathrm{uF}$, the inductance $L_{2}$ is $300 \mathrm{uH}$, the sampling resistance $R_{2}$ is $50 \mathrm{k} \Omega$, the sampling resistance $R_{1}$ is $150 \mathrm{k} \Omega$, the clock signal switching frequency is $20 \mathrm{KHz}$. From the waveform in Fig. 5, it can be seen that the input current tracks the input voltage well, and the input current and input voltage distortion is very small, the overall system power factor is large and system utilization efficiency is high. 


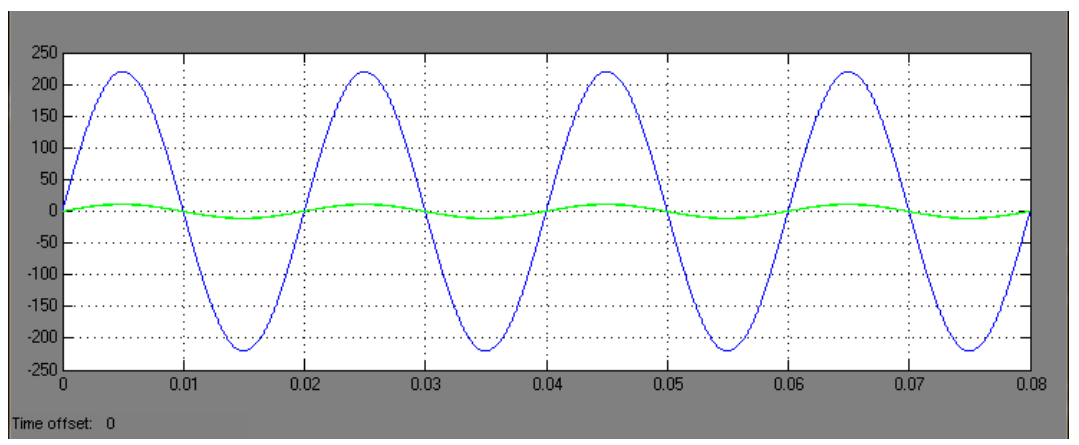

Figure 5. Work waveform of input voltage and current

In order to verify the input interference resistance ability of feedforward control strategy of CUK converter of OCC, in the system input voltage end, superimpose $10 \mathrm{~V}, 100 \mathrm{~Hz}$ sinusoidal signal and $12 \mathrm{~V}, 25 \mathrm{~Hz}$ square wave signal, and then observe the output voltage waveform. As shown in Fig. 6 and Fig. 7, it can be seen that the change of input voltage has no effect on the output voltage waveform, and the dynamic performance of system is very good. It shows that the OCC feedforward control improvement strategy has good input interference resistance ability.

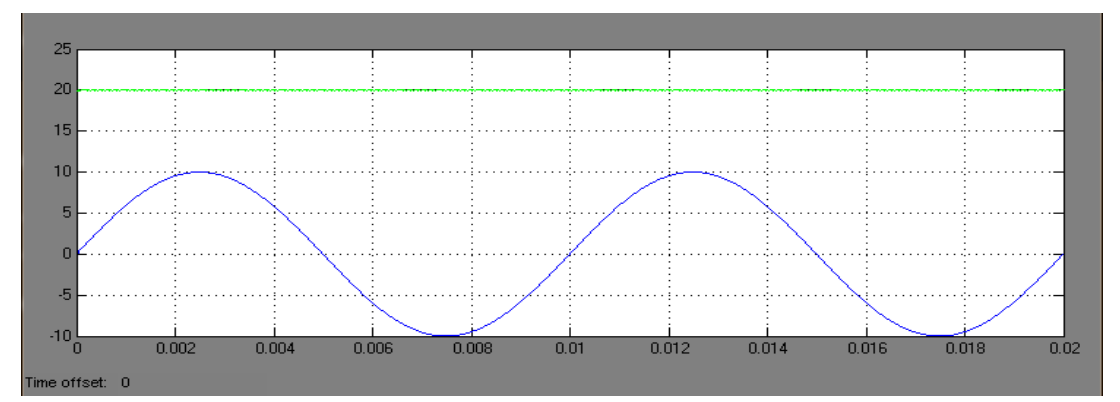

Figure 6. Work waveform under input voltage superposition $10 \mathrm{~V}, 100 \mathrm{~Hz}$ sinusoidal signal

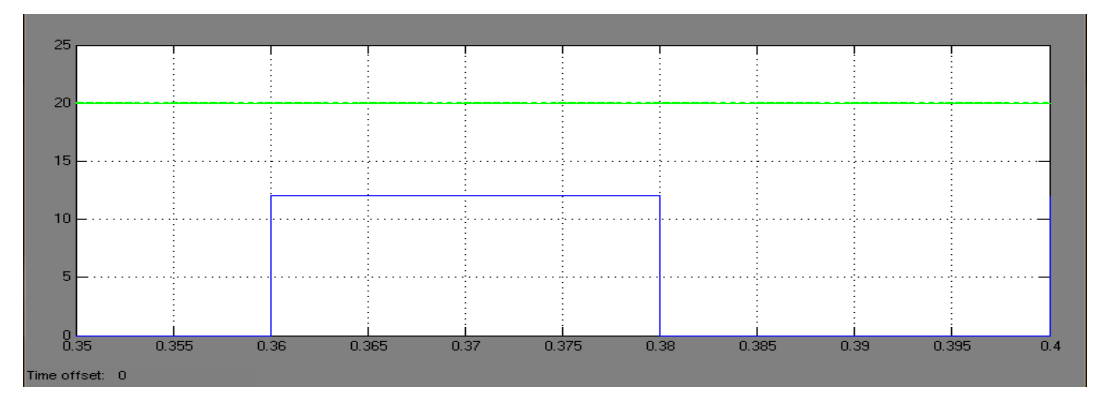

Figure 7. Work waveform under input voltage superposition $12 \mathrm{~V}, 25 \mathrm{~Hz}$ square signal

In order to verify input interference resistance ability of feedforward control strategy of CUK converter of OCC, let the load LED equivalent resistance jump, the load current changes from 1A to 0.8A suddenly. As shown in Fig. 8, the output voltage also has a large mutation, but after a short period, it restores smooth output. We can see OCC feedforward control improvement strategy has a good anti-load interference. 


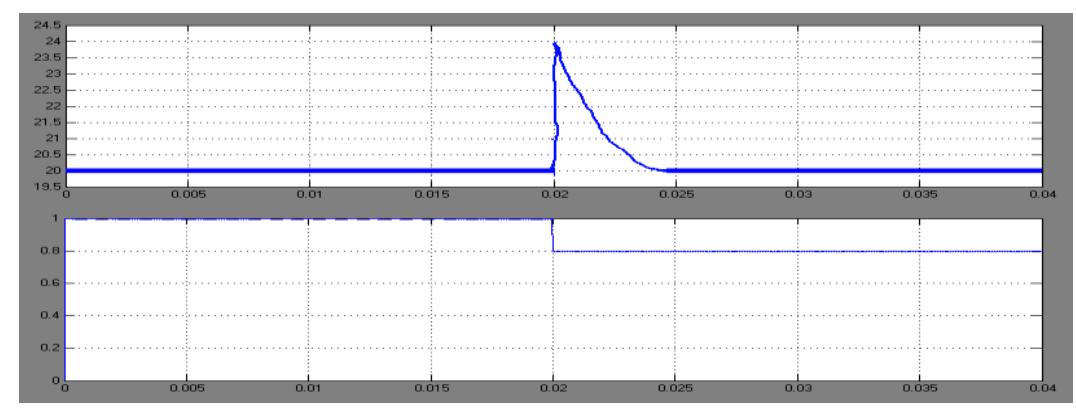

Figure 8. Workflow for load current changing from 1A to $0.8 \mathrm{~A}$

\section{Conclusion}

In this paper, CUK topology-related features are discussed in detail, and a new CUK efficient drive control principle and scheme is proposed, that is, a large-signal nonlinear dynamic control-OCC technique is used to construct the CUK topology semiconductor lighting drive system, SIMULINK and SIMPOWERSYSTEMS software is used to simulate, and the corresponding dynamic relationship expression of the system work is deduced, and the advanced nature of the system, such as fast response, strong anti-interference and good stability are demonstrated.

Funding project: Science Research Key Project of Jiangxi Provincial Education Department (Project No .: GJJ161218).

\section{References}

[1] J.B Xu, J Zhao and L Luo, A new control strategy of unity power factor for three-phase PWM rectifier system. Industrial Electronics Society, 30th Annual Conference of IEEE, 2004, 25(7):709 714

[2] W.K Wu,W.H Q, Efficient high power factor single-stage AC/DC converter. IEEE, 2012, 28(4):1143 1148

[3] X.Q Wu, K Chi and Tse, Fast-Scale Instability of Single-Stage Power-Factor-Correction Power Supplies. IEEE, 2008, 37(5):477 480

[4] J.P Noon, Designing High-Power Factor Off-Line Power Supplies. Proceedings of Unitrode power Supply Design Seminar Manual SEM1500, Texas Instruments, 2007 , 43(2):2 6

[5] E. Najafi, A. Vahedi, A. Mahanfar etal. A new controlling method based on peak current mode(PCM)for PFC. IEEE 2nd International Power and Energy Conference, 2008, 51(3):1103 1107

[6] C.K Tse, Circuit theory of power factor correction in switching converters. Int. j. of Circuit Theory and Applications, 2003, 31(2): 157 198

[7] S Luo, W Qiu A and W Wu, Flyboost power factor correction cell and a new family of single-stage AC/DC converters. IEEE Trans. on Power Electronics, 2011, 20(1): 25 34

[8] P Li, Y.H Yang and H.M Li, APFC control technology for DC energy storage unit of UPQC. Automation of Electric Power Systems, 2009,45(29): 45 48

[9] C Song, J.L Nilles, High-accuracy hysteretic current-mode regulator for powering microprocessors. Applied Power Electronics Conference and Exposition, 2012, 46(5):506 509 
[10] M.K,Ma, X.K Liu, Z Wei and H Zhang, Analysis of fast-scale bifurcations and chaos phenomena in Boost-PFC converter. Proceedings of the CSEE, 2009,39(25): 61 67. 